\title{
Estudio de Clases y sus efectos en las conversaciones pedagógicas docentes
}

\author{
Jorge GAJARDO AGUAYO \\ Jorge ROJAS BRAVO \\ Catherine ALBORNOZ TORRES \\ Javiera ROMERO INOSTROZA
}

Datos de contacto:

Jorge Gajardo Aguayo

Facultad de Educación

Universidad de Concepción, Chile

jorgegajardo@udec.cl

Jorge Rojas Bravo

Escuela de Educación Campus

Los Ángeles

Universidad de Concepción, Chile jorgemanrojas@udec.cl

Catherine Albornoz Torres

Escuela de Educación Campus Los

Ángeles

Universidad de Concepción, Chile catherine.albornoz92@gmail.com

Javiera Romero Inostroza

Escuela de Educación Campus

Los Ángeles

Universidad de Concepción, Chile

javiromero.inostroza@gmail.com

\section{RESUMEN}

Con el propósito de contribuir a la comprensión de los procesos de conversación que se despliegan en el desarrollo de una metodología de desarrollo profesional docente; este estudio describe las modalidades de conversación que emergen en los procesos de planificación colaborativa de dos clases de lenguaje en nivel primario, en el contexto de la implementación de Estudios de Clase. A partir del análisis cualitativo de los turnos completos de palabra en los intercambios docentes y utilizando el modelo de Mercer de análisis de modalidades de conversación (1991), se observa que los docentes participantes transitan desde una modalidad disputativa y acumulativa, hacia una modalidad exploratoria, basada en la colaboración y análisis crítico.

PALABRAS CLAVE: Lesson Study; Desarrollo Profesional Docente; Colaboración; Modalidades de Conversación.

Recibido: 08/11/2020

Aceptado: 10/11/2021 


\title{
Lesson Study and their effects on teaching conversations
}

\begin{abstract}
With the purpose of contributing to the understanding of the conversation processes that are deployed in the development of a methodology for teacher professional development; this study describes the conversation modalities that emerge in the collaborative planning processes of two language classes at the primary level, in the context of the implementation of Lesson Study. From the qualitative analysis of the complete turns of speech in the teacher exchanges and using Mercer's model of analysis of conversation modalities (1991), it is observed that the participating teachers move from a disputative and cumulative modality, to an exploratory modality, based on collaboration and critical analysis.
\end{abstract}

KEYWORDS: Lesson Study; Teaching Professional Development; Collaboration; Conversational Modes.

\section{Introducción}

El presente estudio analiza una experiencia de aprendizaje profesional colaborativo enmarcado en un proceso de desarrollo profesional docente a través del Estudio de Clases (EC). La premisa central que lo orienta es el reconocimiento de que los saberes de los docentes se desarrollan activamente en procesos de intercambio con sus pares, a través de un proceso dialógico.

El EC surge como una herramienta que potencia el desarrollo profesional de los docentes, por medio de colaboración y reflexión entre pares, mitigando el desfase entre lo adquirido en la formación inicial y aquello que los contextos educacionales actuales demandan (Camargo et al., 2004; Osorio, 2016). Estos principios se movilizan en la medida que existan oportunidades para el disenso y la búsqueda de las mejores soluciones a los problemas de práctica que, a través de los intercambios de actores con distintas experiencias y capacidades, genere una visión conjunta de mejora (Calvo, 2014).

Investigar sobre los EC implementados en Chile es significativo para establecer una discusión sobre la importancia de los contextos. Chile presenta una fuerte presión para que los docentes sigan un currículum nacional bastante extenso, donde culturalmente existe una arraigada valoración del trabajo individual (Ávalos \& Bascopé, 2017) y un marco de fuerte competencia entre escuelas bajo un sistema de accountability con altas consecuencias (Parcerisa \& Falabella, 2017). El implementar una metodología con un claro acento hacia el trabajo colaborativo bajo este contexto, presenta un reto que es preciso considerar para el análisis y comprensión de los procesos de desarrollo profesional docente y sus impactos en los aprendizajes de los estudiantes.

Considerando lo anterior, las preguntas de investigación que se abordan son: 
¿Qué modalidad de conversación se promueven entre los y las docentes de segundo año básico que participan en los ciclos de planificación de los EC para el área de lenguaje? ¿Qué cambios en las modalidades de conversación se producen entre los ciclos de planificación de enseñanza, en contextos de trabajo colaborativo como los que provocan los EC?

\section{¿Qué es el Estudio de Clases?}

El Estudio de Clases (EC) es un método de trabajo colaborativo entre docentes, originado en Japón, cuyo objetivo es mejorar las prácticas pedagógicas de los profesores, mientras analizan y critican mutuamente sus métodos y recursos de enseñanza con el fin de impactar positivamente en el aprendizaje de los alumnos, la profesionalización docente, la calidad de la enseñanza y del currículum (Isoda \& Olfos, 2009; Mena, 2007).

Implementar EC aporta beneficios para el desarrollo profesional de los docentes, por cuanto permite la actualización de competencias pedagógicas, disciplinares e investigativas a partir de conversaciones de calidad e indagaciones de los contextos educativos (Elliot, 2015). También los EC permiten que los docentes compartan saberes y estrategias de trabajo con sus pares constituyéndose como un espacio de reflexión para identificar fortalezas y debilidades. Finalmente, los EC permiten el cambio de visión de la enseñanza y el aprendizaje cambiando el foco hacia el aprendizaje de los estudiantes (Soto \& Pérez, 2015; Soto et al., 2019).

Las aplicaciones de los EC fuera de Japón son variadas y con disímiles resultados al considerar el saber acumulado en los contextos (Takashashi \& McDougal, 2016) y los tipos de creencias y culturas escolares presentes (Seleznyov, 2018). En América Latina esta metodología ha comenzado a expandirse gracias a los convenios establecidos con la Agencia de Cooperación internacional de Japón (JICA) (Isoda et al., 2007). En Chile, se han realizado estudios enfocados principalmente al área de matemáticas (Estrella et al., 2020; Garay, 2018; Montoya, 2016; Olfos et al., 2015) y otras recientes exploraciones en el área de la diversidad (Duk \& Hernández, 2020).

Por otro lado, se puede plantear que la lógica de los EC es coherente con las características y desafíos que supone el desarrollo de comunidades profesionales de aprendizaje, en la medida que la colaboración entre el profesorado es un pilar fundamental, que se sostiene en torno al mejoramiento de los aprendizajes de los estudiantes, a través del desarrollo de una cultura de enseñanza basada en la indagación y la mejora de las prácticas de enseñanza (Escudero, 2009; Roberts \& Pruitt, 2009).

El EC se propone como una herramienta para mejorar la enseñanza por medio de la observación y la reflexión docente. Para desarrollar de manera óptima las sesiones del EC se puede invitar a un destacado educador, cuya misión consiste en orientar y entregar los conceptos técnicos y la visión externa sobre el plan de la clase que se desarrolla.

Isoda y Olfos (2009) y Benavides y Calvache (2013), plantean que esta metodología se desarrolla en tres etapas:

A. Preparación: Esta etapa comienza una vez que se ha conformado un grupo de trabajo, el cual puede estar compuesto por docentes de igual o distinto grado, ciclo o asignatura que deseen implementar el EC. El 
proceso se inicia con la revisión de los contenidos a trabajar, se delimita el problema y se establecen los objetivos que se pretenden lograr. El diseño de la clase se realiza teniendo en consideración las dificultades de los estudiantes en torno al objetivo y sus conocimientos previos, las posibles estrategias que se pueden implementar para la clase y los recursos didácticos a utilizar para abordar la temática. En esta fase se debe contar con un formato de plan de clase que guiará el actuar docente.

B. Implementación: Corresponde a la realización de la clase. Previo al inicio de la clase, se les entrega el plan de la lección a los miembros del equipo que participarán como observadores, para que reflexionen en cuanto al desarrollo de la clase. En esta fase se observa el comportamiento de los alumnos, las estrategias metodológicas y uso de recursos del docente. El maestro que dirige la clase tiene pleno conocimiento su estructura y trata de ajustarse a lo planificado.

C. Retroalimentación: tiene como propósito analizar el impacto de la clase en el aprendizaje de los alumnos e identificar los aprendizajes obtenidos para el equipo "Estudio de Clase". El docente realiza una síntesis de lo desarrollado, explica el propósito de la clase y profundiza sobre las decisiones tomadas en lo que se refiere al desarrollo de la clase y la utilización de materiales. Posteriormente se realiza una deconstrucción de la clase, en la cual los miembros del equipo entregan comentarios, formulan preguntas y realizan una reflexión crítica de acuerdo a diversos aspectos de la intervención. Finalmente, a partir de la retroalimentación se realizan ajustes al diseño de la lección con la finalidad generar un mejoramiento progresivo de la enseñanza.

\section{Diálogo y conversaciones profesionales para promover la colaboración y el aprendizaje docente}

El estudio de clases es una instancia de desarrollo profesional docente que contribuye a desarrollar colaboración y reflexión pedagógica. Un elemento clave que promueve el desarrollo de estos principios de aprendizaje, es el diálogo. En efecto, un aspecto esencial de la actividad humana en general se desarrolla bajo condiciones de cooperación e interacción social. Bajo dicha lógica el lenguaje es fundamental, dado que propicia la regulación de la acción y el pensamiento, en la medida que se concibe como un instrumento para realizar distintas acciones (Wells, 2004).

De acuerdo con Mercer (2000) el diálogo puede pensarse, en su especificidad, como una herramienta cultural fundamental, que permite a las personas llegar a una comprensión de los conocimientos y perspectivas de los demás a través del "interpensamiento". De acuerdo con Littleton y Mercer (2013) el diálogo promueve una concepción común de un objetivo compartido, unos conocimientos pertinentes para dicho objetivo y la disponibilidad de un vocabulario de conceptos compartidos que deberían ayudar a los grupos de trabajo profesionales a progresar y desarrollarse. Esto tiene como principio una visión del conocimiento y el aprendizaje como un fenómeno situado (Housley, 2000).

Bajo esta lógica, el aprendizaje profesional promueve un cambio o desarrollo de los conocimientos, los recursos o la comprensión que tienen el potencial de conducir 
a un cambio de comportamiento profesional (Vrikki et al., 2017). Desde una perspectiva sociocultural, se entiende como una construcción conjunta de los participantes en comunidades de práctica, quienes se involucran, a través del diálogo en tareas que requieren de una aproximación colectiva para resolverla (Guerra et al., 2018). Desde esta perspectiva, en la interacción con otros individuos se fomentan procesos cognitivos superiores que favorecen el aprendizaje (Vygotsky, 1978). Por tanto, el desarrollo cognitivo sigue un proceso que transita desde el mundo exterior, social, hacia el mundo interior, individual. Este paso de lo interpersonal a lo intrapersonal es mediado socialmente por distintos instrumentos, entre los cuales el lenguaje y el diálogo, son instrumentos por excelencia (Esteve, 2009). Se puede señalar, en consecuencia, que el habla es la herramienta cultural y psicológica que transforma el pensamiento y acción tanto colectiva como individualmente (Mercer \& Howe, 2012).

El diálogo ofrece tanto un mecanismo de pensamiento y razonamiento crítico, como un marco para la colaboración y la comunicación productivas. Así, una pedagogía dialógica implica a profesores y alumnos en comentar y construir sobre las ideas de cada uno, planteando preguntas y construyendo interpretaciones juntos (Alexander, 2011); "ver" las cosas desde la perspectiva de otro; "encadenar" preguntas y respuestas; y construir y criticar el conocimiento compartido (Mercer, 2000). Este proceso interactivo implica que la acción del participante menos competente es tan importante como la de quien tiene mayor competencia, dado que contribuye a que su interlocutor le dé el soporte necesario, en un proceso de acción conjunta (Esteve, 2009).

No todas las formas de diálogo generan resultados positivos. En grupos de discusión profesionales, las normas sociales implícitas pueden utilizarse para manejar la forma en que se desarrolla el intercambio de conocimiento, particularmente el disenso, generando consensos débiles que no se basan en la mejor solución posible al problema (Littleton \& Mercer, 2013; Vrikki et al., 2017).

Considerando lo anterior, Mercer (2000) distingue entre tres formas de diálogo distintas, que propician de menor a mayor medida la colaboración:

- Conversación de Discusión: Este tipo de conversación se caracteriza por la negativa de los interlocutores a adoptar las ideas del otro, persiste el desacuerdo y la toma de decisiones de forma individual. Los intercambios entre los hablantes son breves en los que predomina la refutación, discusión y afirmación de las ideas. La relación es competitiva, no se comparte información, más bien se expone, generando oposiciones en las diferencias de opinión, promoviendo una posición defensiva. El razonamiento es tácito e individualizado.

- Conversación Acumulativa: En este tipo de conversación, los hablantes abordan de manera positiva, sin criticar las ideas de los demás. Cada uno aporta ideas desde su experiencia y busca enriquecer las opiniones de los demás para construir conocimiento común por medio de la acumulación. Esta conversación es repetitiva, de tipo confirmativo, basada en relaciones implícitas de solidaridad y confianza, requiriendo la confirmación constante de ideas y opiniones.

- Conversación Exploratoria: La característica de este modelo de conversación, es que en ella los interlocutores abordan de manera crítica, pero positiva las 
ideas de los demás. El razonamiento y la justificación son rasgos claves de este tipo de conversación. Esto implica que los argumentos propios tienen a defenderse, mientras los ajenos se cuestionan, generando la necesidad de justificar los elementos sobre los cuales existen dudas, o bien ofreciendo hipótesis alternativas. El progreso en la conversación se manifiesta como el producto de un acuerdo conjunto.

La conversación exploratoria fomenta el desarrollo de patrones colaborativos de trabajo docente, al promover el trabajo hacia metas compartidas. La investigación señala que cuando los miembros de un equipo comparten objetivos de colaboración, participan de intercambios de información más deliberados y exhaustivos, discutiendo directamente sus diferencias de opinión. Esto conduce a niveles más altos de logro de metas y efectividad de grupo (De Dreu, 2007; Johnson \& Johnson, 2005; Tjosvold, 2008). En el caso particular de la conversación exploratoria, diversas investigaciones han demostrado que la discusión abierta de puntos de vista opuestos puede mejorar la calidad de la toma de decisiones en equipo (Lu \& Hallinger, 2017) y la resolución de problemas (Tjosvold, 2008).

Este modelo de conversación que distingue lo disputacional, lo acumulativo y lo exploratorio ha sido utilizado como modelo de análisis de los modos conversacionales en distintos contextos y programas de investigación. Se ha extendido al nivel del diálogo entre estudiantes universitarios (Tan, 2003), estudiantes de pedagogía (Le Cornu \& Ewing, 2008); para analizar los modos conversacionales en el análisis de conflicto y violencia de pareja en adolescentes (Bascón et al., 2013), entre otros.

\section{Metodología}

Con el objetivo de analizar las conversaciones profesionales docentes en el proceso de estudio de clase se utiliza una metodología de corte cualitativa, dado que reconoce la especificidad propia de los contextos e integra a los sujetos como actores relevantes de información y análisis (Mercer, 1991; Strauss \& Corbin, 2002). Considerando la especificidad propia del estudio, el método que se adecua mejor es el método de estudio de caso. Por las características de la investigación se utiliza la modalidad de estudio de caso intrínseco, que se define como el estudio de la particularidad y complejidad de un caso específico para comprender su actividad en circunstancias determinadas (Stake, 1999).

\section{Muestra}

El caso estuvo compuesto por tres escuelas de la ciudad de Concepción, Chile. La Tabla 1 muestra una serie de indicadores de cada escuela. Son instituciones pequeñas, públicas y con alto nivel de vulnerabilidad social de sus estudiantes (IVE). En términos de desempeño académico, la Agencia Nacional de la Calidad de la Educación clasifica a dos de ellas con desempeño Insuficiente. 


\section{Tabla 1}

Caracterización de establecimientos participantes

\begin{tabular}{|c|c|c|c|c|}
\hline Escuela & $\begin{array}{c}\text { Matrícula } \\
2019\end{array}$ & IVE 2019 & $\begin{array}{c}\mathrm{N}^{\circ} \text { Docentes } \\
\text { en la escuela }\end{array}$ & $\begin{array}{c}\text { Categoría de } \\
\text { desempeño }\end{array}$ \\
\hline Escuela 1 & 113 & $93,75 \%$ & 22 & Insuficiente \\
\hline Escuela 2 & 84 & $100 \%$ & 22 & Insuficiente \\
\hline Escuela 3 & 151 & $95,60 \%$ & 26 & Medio \\
\hline
\end{tabular}

Fuente: Tabla de elaboración propia, con datos obtenidos de MINEDUC

Considerando la similitud de su contexto y necesidad de establecer procesos de mejora escolar, se estableció un trabajo en red entre los directivos y docentes. Este estudio recoge el trabajo desarrollado entre los docentes de estas tres instituciones escolares quienes se focalizaron en desarrollar la comprensión lectora de sus estudiantes.

De cada escuela participaron dos docentes: una profesora de educación general básica y otra educadora diferencial, quienes, al momento de desarrollar la investigación, enseñaban en un curso de segundo año de educación general básica. Como profesionales expertos se unieron al proyecto dos profesionales del sistema escolar: una Profesora de Educación General Básica, con mención en lenguaje y jefa de la unidad técnica pedagógica de una escuela y un profesor de lenguaje de enseñanza media, los cuales dieron apoyo disciplinar y pedagógico a los docentes participantes. Este grupo de trabajo fue dirigido por dos académicos universitarios.

Las sesiones programadas para la fase de planificación fueron realizadas en dependencias de la Universidad de Concepción, Chile, por ser considerada una sede neutral para todas las escuelas, lo que permitió que los docentes se desenvolvieran libremente con sus pares. Como medio de información se usaron los registros de audio de las distintas fases desarrolladas del estudio de clase. Estos registros permitieron conocer cómo interactuaron los docentes bajo una nueva instancia de colaboración.

Se consideró como unidad de análisis el turno completo de la palabra y unidad real de la comunicación (Bakhtin, 1986). El análisis se realizó utilizando los modelos de conversación propuestos por Mercer (1991), con el fin de analizar las conversaciones sostenidas por los docentes e identificar extractos de diálogos correspondientes a cada tipo de conversación. Para efectos de este estudio se analizaron los registros de la fase de planificación correspondientes al primer y segundo ciclo de implementación del método EC. Las referencias obtenidas del análisis se añadieron al programa Nvivo12 de acuerdo con cada modelo de conversación, esto nos ayudó a tener una visión general del tipo de conversaciones que sostuvieron los docentes a lo largo del ciclo. Finalmente, se exportó del programa el conteo de las referencias, de acuerdo al tipo de registro y modelo de conversación. 


\section{Resultados}

\section{Modelos de conversación desarrollados en el primer ciclo de EC}

Tras analizar los turnos completos de palabra del primer ciclo de planificación de EC, se identificaron 26 extractos de diálogos que dan cuenta de los modelos de conversación que sostuvieron los docentes en esta instancia. Los resultados obtenidos se resumen en la siguiente tabla.

\section{Tabla 2}

Modelos de Conversación en primer ciclo de planificación, escuela 1

\begin{tabular}{|l|c|}
\hline Modelo de Conversación & Número de referencias \\
\hline Conversación de Discusión & 8 \\
Conversación Acumulativa & 12 \\
Conversación Exploratoria & 6 \\
Total & 26 \\
\hline
\end{tabular}

Fuente: Tabla de elaboración propia

De acuerdo con los datos presentes en la tabla, se describen a continuación los modelos de conversación según la frecuencia con la que se dieron durante el ciclo y se describen de acuerdo con la presencia que estos tuvieron en cada registro:

\section{Conversación de Discusión}

De acuerdo con la información presente en la tabla 2, las conversaciones de discusiones estuvieron presentes en 8 oportunidades. En estas conversaciones los docentes escuchaban las opiniones de sus compañeros, pero no las integraban a su visión para construir conocimiento; más bien, constantemente reafirmaban sus propias opiniones. Las discusiones establecidas por los docentes se dieron en torno al esquema de la planificación utilizada, las actividades a desarrollar en torno al objetivo propuesto y las adecuaciones curriculares (Ver ejemplo 1):

\section{Ejemplo 1}

Docente 1: Pero yo encuentro que le están dando muchas vueltas, les están dando muchas facilidades a ellos.

Docente 5: No, pero es que eso es considerando a los que no leen, a esos nos estamos enfocando también, para que ellos comprendan lo mismo que los que leen.

Docente 6: Claro, si incluso los avanzados van a ir ayudando a los que están más atrás.

Docente 7: Pero al momento de, ellos van a tener una actividad diferente.

En el ejemplo anterior, se establece una relación competitiva entre los docentes que gira en torno a la posición y rol que se les asignará a los estudiantes en el 
desarrollo de las actividades. Se evidencian al menos tres posiciones contrarias y defensivas: sobre el esfuerzo del estudiante, sobre los estudiantes según su nivel de desarrollo de habilidades y sobre el rol del estudiante.

\section{Conversación de Acumulación}

Los resultados generales de la tabla 2 nos indica que el modelo de conversación que se dio con mayor frecuencia entre los docentes a lo largo del ciclo fue la conversación de tipo acumulativa con un total de 12 referencias, lo que nos indica que la mayor parte del tiempo los docentes sostuvieron conversaciones de manera positiva, en las cuales cada uno aportó información en base a su experiencia con la finalidad de construir conocimiento compartido. Los diálogos sostenidos por los docentes durante esta etapa se orientaron en torno a las actividades y las estrategias a utilizar, las adecuaciones curriculares (Ejemplo 2) y los tiempos de cada fase de la clase:

\section{Ejemplo 2}

Docente 2: ¿Si trabajamos los pictogramas?, dibujos y lectura, se van cambiando por imágenes los personajes, entonces así van leyendo eso... es bueno igual, porque ellos lo van a ver y van a ir leyendo igual que sus compañeros, se les puede entregar a todos de hecho, porque va lectura más imágenes.

Docente 5: Esa es la gracia de la clase, que sea pareja para todos.

Docente 6: A veces hay textos en los que sale el dibujo del animal en vez del nombre, entonces ellos leen el ratón fue... y sale no sé, comer y sale un queso.

Docente 1: Cómo podemos representar toda esa lectura así, tendría que ser algún extracto de la lectura, leerlo para todos, que ellos participen y después alguna actividad más pequeña tomar alguna frase, hacerlos que la lean, por ejemplo: el león. Ellos deberían ser capaces de leerlo y trabajar eso con ellos que logren hacer la decodificación más que nada, es que claro, por ejemplo, el Dante reconoce la L y la D porque él se llama Luis Dante y todos le dicen Dante.

En el ejemplo anterior, la conversación de los docentes asume una posición afirmativa, que carece de controversia y acumula opiniones sobre el desarrollo de una actividad de aprendizaje. Los docentes no abordan abiertamente una disputa, aún cuando sus opiniones no son del todo coherentes. Esto se observa respecto de lo planteado por el docente 6, frente a lo señalado por el docente 2 . La divergencia se manifiesta latente, sin escalar ni aportar al desarrollo de una actividad más robusta que aborde el problema de aprendizaje de los no-lectores y facilite el desarrollo de habilidades de los lectores.

\section{Conversación exploratoria}

Este tipo de conversación fue el que se dio con menos frecuencia entre los docentes según la tabla 2. De los tres registros analizados solo se logró extraer 6 referencias relacionadas a la conversación exploratoria, lo que refleja que a los 
enseñantes se les dificulta criticar de manera constructiva, entregar fundamentos sobre sus opiniones y analizar distintos puntos de vista para progresar en conjunto.

Este tipo de conversaciones se desarrollaron solamente durante la etapa de planificación. Las referencias obtenidas de este registro nos indican que los diálogos establecidos por los docentes se centraron en diseñar y revisar el material que se utilizaría para la clase (Ejemplo 3), organizar las actividades en relación con la secuencia de la clase, evaluar y modificar el objetivo de aprendizaje.

\section{Ejemplo 3}

Docente 6: Esa va a ser la pregunta, ¿De qué cree que tratará la clase de hoy, mirando las imágenes? Entonces, alguno va a decir a lo mejor que, sobre un cuento o algo ya visto anteriormente.

Docente 5: Pueden decir algo relacionado con los personajes.

Docente 6: Y con eso nomás, porque ahí se van a explayar y después comienza la actividad.

Docente 7: ¿Cómo se dieron cuenta de eso? Para retroalimentar.

Docente 6: Es que, para después, eh ya aquí después de leer el texto, entonces ahí después se les pregunta qué tipo de texto es.

Docente 7: No, la imagen primero yo creo, es que, para vincularlos altiro desde el inicio con esta habilidad, el título, presentarles primero la imagen y después el título y ahí ¿De qué se tratará?, no de un león un ratón ¿Por qué crees tú eso? A lo mejor alguno ya lo conoce.

En el ejemplo anterior, se evidencia una relación de colaboración efectiva, en la medida que frente a la propuesta de actividades se desarrollan procesos de controversia, que se abordan desarrollando procesos de razonamiento y justificación. Frente a la propuesta de actividad de aprendizaje (docente 6), se comienzan a desarrollar distintos escenarios, los que son puestos a prueba por los docentes (docente 7), de tal modo que se logra desarrollar una actividad de aprendizaje más robusta.

\section{Modelos de Conversación desarrollado en segundo ciclo de EC}

Considerando las conversaciones establecidas en el primer ciclo de planificación, la tabla 3 nos evidencia los cambios que se registraron en el segundo ciclo de planificación. El propósito de haber realizado este análisis fue establecer una comparación entre los registros de ambas planificaciones con el fin de identificar si existió una variación en las conversaciones, para establecer desde la lógica de Mercer, si los diálogos desarrollados por los docentes transitaron desde una conversación de discusión hacia una conversación exploratoria. Del análisis de este registro se obtuvieron 15 extractos de diálogos, los cuales de acuerdo a los diferentes modelos de conversación se clasifican en la siguiente tabla: 


\section{Tabla 3}

Modelos de Conversación en segundo ciclo de planificación, escuela 2

\begin{tabular}{|c|c|}
\hline Modelo de Conversación & Número de referencias \\
\hline Conversación de Discusión & 0 \\
Conversación Acumulativa & 5 \\
Conversación Exploratoria & 10 \\
Total & 15 \\
\hline
\end{tabular}

Fuente: Tabla de elaboración propia

\section{Conversación de Discusión}

En este registro no fue posible evidenciar extractos de conversaciones de este modelo.

\section{Conversación Acumulativa}

De acuerdo con la información contenida en la tabla 3, se obtuvieron cinco extractos de conversaciones de tipo acumulativas. Estas se desarrollaron en torno al vocabulario de los alumnos, el cumplimiento del objetivo en la alumna con necesidades educativas, mejoramiento del manejo de aula (Ejemplo 4) y confianza de los alumnos.

\section{Ejemplo 4}

Docente 7: Por eso te preguntaba cómo tú veías tú pedagogía a futuro, si eso es parte de tu manera de enseñar.

Docente 2: Es que no sé, a veces el resto como que se espanta.

Docente 8: Que esto igual es como un desafío personal, el hacia donde quiero moverme, tú necesitas a los niños más estructurados o ¿no?

Docente 2: Es que depende de la situación.

Docente 1: Para que escuchen lo que tiene que hacer, por ejemplo, cuando están haciendo algo, porque se ponen así como abejas y suben bajan algunos se cambian.

Docente 2: Sí.

Docente 6: Eso es lo que me pasa a mí, yo quisiera que los niños me escucharan, se quedaran quietos y en silencio y no volara ni una mosca, pero no puedo porque ellos no me dejan.

En el ejemplo anterior, la conversación que sostienen los docentes gira en torno a la identidad profesional y su visión de aprendizaje y enseñanza. No obstante, no se abren disputas ni controversias. Existe una aceptación acrítica de la opinión de los demás (docente 2), aún cuando tácitamente se manifiestan ciertas diferencias respecto del rol de los estudiantes y su relación con el docente, ya sea aludiendo a la flexibilidad del rol (docente 8 y 1), frente a un rol pasivo del estudiante (docente 6). 


\section{Conversación Exploratoria}

En relación a la información presentada en la tabla 3, durante esta fase el modelo de conversación exploratoria fue el que se dio con mayor frecuencia. Las conversaciones se centraron en temas como: estrategias para el desarrollo del vocabulario, tipo de texto a utilizar, actitudes a desarrollar en la clase, adecuación de las actividades en torno al tiempo, clasificación de los alumnos según el desarrollo de la habilidad, estrategias para manejo de aula, construcción del objetivo (Ejemplo 5) y la estrategia para el desarrollo del objetivo.

\section{Ejemplo 5}

Docente 3: Es que para elegir uno de esos deberíamos tener el objetivo de la clase, ver qué tipo de texto queremos trabajar para elegir uno de esos.

Docente 7: No profesora, se supone que es al revés.

Docente 8: Para poder determinar el objetivo se debe tener claridad en lo que pretendo lograr exactamente, por eso estamos viendo esto para ver qué exactamente de reflexionar vamos a ver, vamos a emitir un juicio de valores o una lectura crítica, en que nos vamos a enfocar. Tenemos que ver que más que sea algo entretenido que se cumpla el objetivo al final de la clase.

Docente 3: A nosotros nos pasó eso que al final se perdió el foco en la actividad.

Docente 7: Recuerda que uno de los objetivos de esa clase era que el alumno dijese que había hecho tal cosa, entonces ahí está nuestro mayor indicador de logro.

En el ejemplo anterior, se observa una disputa que origina una reconfiguración de una posición discursiva, en la medida que se adopta el punto de vista del docente que abre la controversia. Mientras el docente 3 plantea una estrategia para determinar el objetivo de la clase, es abiertamente refutado por los docentes 7 y 8 , quienes aportan evidencia sobre un punto de vista diferente sobre el diseño de objetivos de clase. Esto lleva al docente 3 a plantear evidencia sobre su propia experiencia, que refuerza lo planteado por sus colegas.

\section{Cambio y transición en los modelos de conversación}

Del análisis de ambas planificaciones se pudo evidenciar que las conversaciones sostenidas por los docentes fueron variando de un ciclo a otro. Los resultados obtenidos de la primera planificación nos indicaron que el modelo de conversación que se dio con mayor frecuencia fue la conversación acumulativa. En cambio, durante la segunda planificación el modelo de conversación que más se destacó fue la conversación exploratoria.

Al contrastar estos resultados se concluyó que a medida que el proyecto fue avanzando, las formas de conversar entre los docentes fueron variando. En un inicio las conversaciones estuvieron basadas en la construcción positiva y conjunta, pero desde una lógica acumulativa donde las ideas aportadas por cada miembro del equipo fueron aceptadas sin que existiera un mayor análisis.

Posteriormente una vez que los docentes adquirieron mayor confianza para trabajar en equipo, las conversaciones que se generaron entre ellos se 
fundamentaron en una construcción positiva, pero crítica del conocimiento. Las aportaciones realizadas fueron analizadas, debatidas y evaluadas por todo el equipo, lo que evidenció un mayor razonamiento por parte de los docentes en la toma de decisiones, con diálogos más largos y sostenidos en el tiempo.

Según Mercer (1997), las conversaciones de tipo exploratoria son aquellas a las que se aspira llegar en una conversación educativa. Este tipo de conversaciones ayuda a desarrollar hábitos intelectuales, ya que implica un mayor razonamiento y análisis por parte de los sujetos, por ende, es la más efectiva de los tres modelos de conversación para resolver problemas a través de la actividad colaborativa.

No obstante, llama la atención que el contenido de los tópicos abordados en las conversaciones disputativas del primer ciclo y en las acumulativas del segundo ciclo, se refieren principalmente al rol de los estudiantes y su conducta. Un elemento clave que podría explicar esto dice relación con las diferencias asociadas a las visiones de aprendizaje de cada docente (Gajardo, 2019) y sus teorías implícitas sobre el aprendizaje (Pozo et al. 2006). Precisamente, se logra transitar desde lo disputativo a lo exploratorio, en la medida que los tópicos abordan con mayor profundidad los elementos técnicos asociados al desarrollo de actividades, las estrategias y materiales a utilizar. Pero en los turnos completos de palabras, los tópicos que hacen referencia a la visibilización del estudiante en su contexto y considerando sus desafíos de aprendizaje, se vinculan a distintas visiones que contrastan y no son fáciles de abordar ni resolver.

\section{Discusión y conclusiones}

El trabajo colaborativo entre docentes propicia el desarrollo de intercambios pedagógicos que facilitan el aprendizaje compartido. El aprendizaje colaborativo es uno de los enfoques principales en materia de desarrollo profesional (Vaillant, 2016), pero dificultado por la presencia de una cultura individualista de trabajo (Arévalo \& Núñez, 2016). Uno de nuestros hallazgos, sugiere que producto del trabajo individualista existen habilidades que se encuentran disminuidas en los docentes, como el escuchar sugerencias y aceptar opiniones de otros. En este sentido, propiciar un espacio de colaboración entre iguales, permite a los docentes aprender de los otros y crecer profesionalmente. Tal como indica Soto, Serván y Peña (2019), el carácter colaborativo del EC es lo que posibilita a los docentes tomar conciencia sobre la importancia de trabajar en equipo.

Los resultados del análisis de las conversaciones aportan en dicho sentido, en la medida que los docentes transitan desde una posición centrada en la discusión y disputa, hacia la participación en diálogos exploratorios, de tipo colaborativo en lugar de competitivo. En efecto, los diálogos centrados en la discusión asumen la forma de una competencia, que implica la presencia de un "ganador" (Wegerif \& Mercer, 1997). Esto tiene diversas consecuencias para el proceso de aprendizaje profesional. En primer lugar, los focos de las discusiones se mueven hacia aspectos más profundos de la dinámica del aprendizaje, con una mayor responsabilización de los aprendizajes de los estudiantes. Del mismo modo, las conversaciones son más extensas y técnicas desde un punto de vista pedagógico, abriendo camino a un plano mucho más reflexivo, que requiere la justificación de hipótesis y su revisión permanente. 
Lo anterior es coherente con las concepciones actuales de aprendizaje y desarrollo profesional docente, en la medida que el conocimiento se integra a las experiencias profesionales de los docentes, como resultado de su participación activa reflexionando sobre su práctica y modificando su enseñanza para promover un mejor aprendizaje de los estudiantes (Vescio et al., 2008). Asimismo, promueve y es coherente con el desarrollo de una comunidad profesional de aprendizaje, en la medida que propicia el desarrollo de relaciones de colaboración entre el profesorado, desarrollando procesos de investigación sobre y de la práctica (Escudero, 2009; Roberts \& Pruitt, 2009).

La participación de profesores de distintos centros escolares es un factor importante para considerar en la implementación de EC en el contexto nacional, en la medida que genera condiciones que facilitan el tránsito desde diálogos acumulativos hacia conversaciones exploratorias. La heterogeneidad de prácticas y visiones presente en los docentes es una oportunidad, dado que los participantes no comparten un espacio formativo y una cultura escolar que promueva ideas similares entre ellos (Guerra et al., 2018). Bajo ciertas condiciones, esto promueve el disenso, necesario para la integración de nuevas ideas, como las que se asocian, por ejemplo, a la visión de aprendizaje y las creencias tácitas sobre el aprender. Cuando los contenidos de las conversaciones gravitaban en torno a dichos aspectos, era más complejo avanzar hacia una conversación de tipo exploratorio.

\section{Limitaciones}

Se reconoce como limitación del estudio lo acotado de la muestra y la imposibilidad de completar los tres ciclos comprometidos. Producto de las contingencias sociales vividas en Chile durante el año 2019, los resultados expuestos dieron cuenta del trabajo de dos de las tres escuelas participantes del proyecto.

\section{Referencias}

Alexander, R. (2011). Towards dialogic teaching: Rethinking classroom talk. Dialogos. Arévalo, A. y Núñez, M. (2016). Los Profesores Hablan. Docencia, (60), 55-65.

Ávalos, B. y Bascopé, M. (2017). Teacher informal collaboration for professional improvement: beliefs, contexts, and experience. Education Research International, 1-13. https://doi.org/10.1155/2017/1357180

Bakhtin, M. (1986). Speech genres and other late essays. University of Texas Press.

Bascón, M. J., Arias, S. y de la Mata, M. L. (2013). Contenidos y modos conversacionales en adolescentes. Debatiendo sobre conflictos grupales y violencia de pareja. $\begin{array}{llll}\text { Infancia } y & \text { Aprendizaje, } & \text { 36(4), }\end{array}$ https://doi.org/10.1174/021037013808200302

Benavides, L. y Calvache, R. (2013). El Estudio de Clase Como Investigación en el Aula. Docencia, Investigación e Innovación, 2(1), 32-55.

Calvo, G. (2014). Desarrollo Profesional Docente: El Aprendizaje Profesional Colaborativo. En UNESCO (Ed.), Temas críticos para formular nuevas políticas docentes en América Latina y el Caribe. El debate actual (112-152). Ceppe y Unesco.

Camargo, M., Calvo, G., Franco, M., Vergara, M., Londoño, S., Zapata, F. y Garavito, 
C. (2004). Las necesidades de Formación Permanente del Docente. Educación y educadores, 7(1), 79-112.

De Dreu, C. K. W. (2007). Cooperative outcome interdependence, task reflexivity, and team effectiveness: A motivated information processing perspective. Journal of Applied Psychology, 92, 628-638. doi:10.1037/00219010.92.3.628

Duk, C. y Hernández, F. (2020). Mejorando la Respuesta a la Diversidad en el Aula a través del Estudio de Clases en Escuelas Chilenas. Revista Internacional de Educación para la Justicia Social, 9(1), 99-123. https://doi.org/10.15366/riejs2020.9.1.005

Elliot, J. (2015) Lesson y learning Study y la idea del docente como investigador. Revista Interuniversitaria de Formación del Profesorado, 29 (3), 29-46.

Escudero, J. M. (2009). Comunidades docentes de aprendizaje, formación del profesorado y mejora de la educación. Ágora Para La Educación Física y El Deporte, 10, 7-31. http://hdl.handle.net/11162/24566

Estrella, S., Zakaryan, D., Olfos, R. y Espinoza, G. (2020). How teachers learn to maintain the cognitive demand of tasks through Lesson Study. Journal of Mathematics Teacher Education 23, 293-310 https://doi.org/10.1007/s10857-018-09423-y

Esteve, O. (2009). La interacción, un proceso que implica conversar. Cuadernos de Pedagogía, (391), 56-59.

Gajardo, J. (2019). Analizando la visión de aprendizaje presente en el establecimiento desde un enfoque de Aprendizaje Profundo. Nota Técnica No 6. LIDERES EDUCATIVOS, Centro de Liderazgo para la Mejora Escolar.

Garay, S. (2018). De Japón a Puente Alto: El estudio de la clase: una innovación en la enseñanza de las matemáticas. Revista de Gestión de la innovación de Educación Superior REGIES, 3(1), 94-114.

Guerra, P., Grau, V., Sebastián, C., y Galdames, A. (2018). Análisis de las interacciones entre estudiantes de pedagogía durante un juego serio. Revista de Psicología, 27(1), 1-13. https://doi.org/10.5354/0719$\underline{0581.2018 .50750}$

Housley, W. (2000). Category work and knowledgeability within multidisciplinary team meetings. Text \& Talk, 20(1), 83-107. https://doi.org/10.1515/text.1.2000.20.1.83

Isoda, M., Arcavi, A. y Mena, A. (2007). El Estudio de Clases Japonés en Matemáticas: Su importancia para el mejoramiento de los aprendizajes en el escenario global. Ediciones Universitarias de Valparaíso.

Isoda, M. y Olfos, R. (2009). El Enfoque de Resolución de Problemas, en la enseñanza de la matemática a partir del estudio de clases. Ediciones Universitarias de Valparaíso.

Johnson, D. W., \& Johnson, R. T. (2005). New developments in social interdependence theory. Genetic, Social, and General Psychology Monographs, 131, 285-358. doi:10.3200/ MON0.131.4.285-358

Le Cornu, R. \& Ewing, R. (2008). Reconceptualising professional experiences in pre-service teacher education... reconstructing the past to embrace the future. Teaching and Teacher Education, 24(7), 1799-1812. https://doi.org/10.1016/j.tate.2008.02.008 
Littleton, K., \& Mercer, N. (2013). Interthinking: Putting talk to work. London: Routledge.

Lu, J., \& Hallinger, P. (2017). A Mirroring Process: From School Management Team Cooperation to Teacher Collaboration. Leadership and Policy in Schools, $17(2)$ 238-263. https://doi.org/10.1080/15700763.2016.1278242

Mena, A. (2007). El Estudio de Clases japonés en perspectiva. Informe de investigación presentado en la XIII Jornada de la Sociedad Chilena de Educación Matemática. Pontificia Universidad Católica de Valparaíso, Chile. Recuperado de http://mastecnica.cl/?mdocs-file=1135

Mercer, N. (1991). Investigaciones sobre el conocimiento compartido. En G. Walford. (Ed.), La otra cara de la investigación educativa (59-78). Editorial La Muralla.

Mercer, N. (1997). La construcción guiada del conocimiento. El habla de profesores y alumnos. Ediciones Paidós Ibérica.

Mercer, N. (2000). Words and Minds: How We Use Language to Think Together. Routledge.

Mercer, N., \& Howe, C. (2012). Explaining the dialogic processes of teaching and learning: The value of sociocultural theory. Learning, Culture and Social Interaction, 1(1), 12-21. https://doi.org/10.1016/j.lcsi.2012.03.001

Montoya, M. (2016). Reflexiones de profesores en un escenario de Estudio de Clases para el desarrollo profesional. Estudios Pedagógicos, 42(4), 127-144. http://dx.doi.org/10.4067/S0718-07052016000500008

Olfos, R., Estrella, S., y Morales, S. (2015). Clase pública de un estudio de clases de estadística: una instancia de cambio de creencias en los profesores. Revista Electrónica Educare, 19(3), 1-17. doi: http://dx.doi.org/10.15359/ree.19-3.21

Osorio, A. (2016). El Desarrollo Profesional Docente en Educación Básica Primaria. Revista Latinoamericana de Estudio Educativo, 12(1), 39-52.

Parcerisa, L. y Falabella, A. (2017). La consolidación del Estado Evaluador a través de políticas de rendición de cuentas: trayectoria, producción y tensiones en el Sistema educativo chileno. Education Policy Analysis Archives, 25(89). http://dx.doi.org/10.14507/epaa.25.3177

Pozo, J. I., Scheuer, N., Pérez, M., Mateos, M., Martín, E., y de la Cruz, M. (2006). Nuevas formas de pensar la enseñanza y el aprendizaje. Las concepciones de profesores y alumnos. Barcelona: Editorial GRAÓ.

Roberts, S. \& Pruitt, E. (2009). Schools as Professional Learning Communities. Corwin Press.

Seleznyov, S. (2018). Lesson study: an exploration of its translation beyond Japan. International Journal for Lesson and Learning Studies, 7 (3), 217-229.

Soto, E. y Pérez, A. (2015). Lesson Studies: Un viaje de ida y vuelta recreando el aprendizaje comprensivo. Revista Interuniversitaria de Formación del Profesorado, 29 (3), 15-28.

Soto, E., Serván, M.J., Peña, N. y Pérez-Gómez, Á. I. (2019). Nuevos retos en la formación del profesorado. Lesson Study: acompañar la enseñanza y la investigación. Márgenes, Revista de Educación de la Universidad de Málaga, 0(0), 38-57. https://doi.org/10.24310/mgnmar.v0i0.6504

Stake, R. (1999). Investigación con Estudio de Caso. Ediciones Morata, L.S.

162 ISSN 0213-8646 | E-ISSN 2530-3791 • Revista Interuniversitaria de Formación delProfesorado, 96 (35.3) (2021), 147-164 
Strauss, A. L. \& Corbin, J. (2002). Bases de la investigación cualitativa: técnicas y procedimientos para desarrollar la teoría fundamentada. Editorial Universidad de Antioquia.

Takahashi, A., \& McDougal, T. (2016). Collaborative lesson research: maximizing the impact of lesson study. ZDM Mathematics Education, 4, 513-526.

Tan, B. T. (2003). Does talking with peers help learning? The role of expertise and talk in convergent group discussion tasks. Journal of English for Academic Purposes, 2(1), 53-66. https://doi.org/10.1016/S1475-1585(02)00033-4

Tjosvold, D. (2008). Controversy for management education: Developing committed, open- minded researchers. Exemplary contributions. Academy of Management Learning and Education, 7, 73-85. doi:10.5465/AMLE.2008.31413864

Vaillant, D. (2016). Trabajo colaborativo y nuevos escenarios para el desarrollo profesional docente. Docencia, (60), 5-13.

Vescio, V., Ross, D. y Adams, A. (2008). A review of research on the impact of professional learning communities on teaching practice and student learning. Teaching and Teacher Education, 24, 80- 91. https://doi.org/10.1016/j.tate.2007.01.004

Vrikki, M., Warwick, P., Vermunt, J. D., Mercer, N., \& Van Halem, N. (2017). Teacher learning in the context of Lesson Study: A video-based analysis of teacher discussions. Teaching and Teacher Education, 61, 211-224. https://doi.org/10.1016/j.tate.2016.10.014

Vygotsky, L. S. (1978). Mind in Society: the De velopment of Higher Psychological Processes. Harvard University Press.

Wegerif, R. y Mercer, N. (1997). A dialogical framework for researching peer talk. In Wegerif, R. y Scrimshaw, P. (Eds.), Computers and talk in theprimary class room (pp. 49-61). Multilingual Matters.

Wells, G. (2004). El papel de la actividad en el desarrollo y la educación. Infancia $y \quad$ Aprendizaje, 27(2), 165-187. https://doi.org/10.1174/021037004323038824 
164 ISSN 0213-8646 | E-ISSN 2530-3791 • RevistaInteruniversitaria de Formación delProfesorado, 96 (35.3) (2021), 147-164 